\title{
Identification of differentially expressed microRNAs in metastatic melanoma using next-generation sequencing technology
}

\author{
MIN QI $^{1}$, XIAOYUAN HUANG ${ }^{1}$, LEI ZHOU ${ }^{2}$ and JIANGLIN ZHANG ${ }^{2}$ \\ Departments of ${ }^{1}$ Plastic Surgery and ${ }^{2}$ Dermatology, Xiangya Hospital, \\ Central South University, Changsha, Hunan 410008, P.R. China
}

Received November 14, 2013; Accepted February 20, 2014

DOI: $10.3892 /$ ijmm.2014.1668

\begin{abstract}
In this study, we investigated differentially expressed microRNAs (miRNAs or miRs) and their functions in metastatic melanoma using next-generation sequencing technology. The GSE36236 data set was downloaded from the Gene Expression Omnibus (GEO) database and 4 primary cutaneous melanoma samples (used as controls) and 3 metastatic melanoma samples were selected from 31 samples for further analysis. Firstly, the differentially expressed miRNAs were screened by limma package in $\mathrm{R}$ language. Secondly, the target genes of the miRNAs were retrieved with TargetScanHuman 6.2, and the interactions among these genes were identified by String and an interaction network was established. Finally, functional and pathway analyses were performed for the genes in the network using Expression Analysis Systematic Explorer (EASE). A total of 4 differentially expressed miRNAs (hsa-miR-146, hsa-miR-27, hsa-miR-877 and hsa-miR-186) were obtained between the metastatic melanoma and primary cutaneous melanoma samples. We predicted 101 high-confidence target genes of hsa-miR-27 and obtained a network with 41 interactions. Finally, functional and pathway analyses revealed that the genes in the network were significantly enriched at the transcriptional level. Differentially expressed miRNAs were identified in the metastatic melanoma compared with the primary cutaneous melanoma samples and the target genes of hsa-miR-27 were found to be significantly enriched at the transcriptional level. The results presented in our study may prove helpful in the diagnosis and treatment of metastatic melanoma.
\end{abstract}

\section{Introduction}

The incidence of metastatic melanoma and mortality rate has continually increased over the past decades $(1,2)$. The invasive and metastatic ability are the most fundamental features of

Correspondence to: Dr Jianglin Zhang, Department of Dermatology, Xiangya Hospital, Central South University, 87 Xiangya Road, Changsha, Hunan 410008, P.R. China

E-mail: jianglinzhang2@hotmail.com

Keywords: metastatic melanoma, differentially expressed microRNAs, target gene prediction, interaction network, enrichment analysis cancer cells which differ from normal cells, eventually leading to tumor recurrence, progression and ultimately, death (3). The median survival of patients with metastatic melanoma is $<1$ year (4). Thus, an improvement in therapy for metastatic melanoma is mandatory.

A number of studies have revealed that microRNAs (miRNAs or miRs) are associated with the metastasis of colon cancer, breast cancer and lung cancer cells (5-7). Over the past few years, the association between miRNAs and tumors has become a research hotspot. Certain miRNAs have been proven to be potential biomarkers in clinical diagnosis $(8,9)$, which have the most extensive gene regulation functions on several levels (10) and are involved in a series of important biological processes, including early embryonic development, cell proliferation, apoptosis, cell death and fat metabolism, and even the differentiation of stem cells (11). Due to the advances in miRNA research, an increasing number of studies have demonstrated that miRNAs regulate important tumor-related genes, and are thus closely related to the development and occurrence of tumors. For example, miR-7 and miR-331-3p have been shown to regulate the expression of epidermal growth factor receptor (EGFR) and human EGFR, which are biomarkers of human tumors (12). Jiang et al demonstrated that miRNA-155 acts as a suppressor of the cytokine signaling 1 gene that is a tumor suppressor in breast cancer (13). However, a number of tumor-associated miRNAs remain unexploited, and the mechanisms responsible for the regulatory functions of miRNAs in metastatic melanoma are not yet fully understood. Therefore, miRNAs may provide a novel treatment method for cancers.

Given the detrimental effects of metastatic tumors and the growing development of sequencing technology, small RNA sequencing was adopted in our study to identify the differentially expressed miRNAs in metastatic melanoma compared with primary cutaneous melanoma samples. The target genes were predicted, and functional and pathway analyses were performed to reveal their roles in the progression of metastasis. Our findings may provide new insight into the diagnosis and treatment of metastatic tumors.

\section{Materials and methods}

Sequencing data. The GSE3623 sequencing data, including a total of 31 samples, was downloaded from the Gene Expression 
Table I. Statistical information of the sequencing data.

\begin{tabular}{lcccc}
\hline Group & Accession no. & No. of original reads & No. of miRNA alignments & No. of miRNA types \\
\hline Metastatic melanoma & GSM893568 & 402556 & 17847 & 259 \\
& GSM893576 & 1226137 & 18005 & 123 \\
& GSM893577 & 708653 & 16493 & 262 \\
Primary melanoma & GSM893578 & 395686 & 31687 & 279 \\
& GSM893564 & 97403 & 85049 & 338 \\
& GSM893566 & 464804 & 40816 & 40267 \\
\hline
\end{tabular}

Omnibus (GEO) database. A total of 7 melanoma samples were selected, including 4 primary cutaneous melanoma samples (used as controls) and 3 metastatic melanoma samples. Raw data were obtained in SRA format, as previously described (14). The platform used was GPL9052 Illumina Genome Analyzer (Homo sapiens). Deep sequencing was performed on various tissues and sequencing data were obtained. We transformed the data from SRA format to FASTQ format, as previously described (15) using the SRA Toolkit (16). Sequence alignments were then carried out with Bowtie alignments (17), allowing as many as 2 mismatches. Bowtie is a rapid and memory-saving tool for short sequence assembly. It aligns short sequences to reference genomes and can read base pairs as long as $1024 \mathrm{bp}$, which renders it extremely suitable for nextgeneration sequencing technologies (18). The expression level of miRNAs was determined using miRDeep, as previously described (19), which has been proven to be of high sensitivity and accuracy (20).

Screening of differentially expressed miRNAs. After the expression levels were determined, the differentially expressed miRNAs were screened out between the metastatic melanoma and primary cutaneous melanoma (controls) samples with limma package in $\mathrm{R}$ language (21); a p-value $<0.05$ and $|\log \mathrm{FC}|>1$ were set as the cut-off values.

Prediction of target genes of miRNAs. Target genes were predicted using TargetScanHuman 6.2, which is a software package developed by Lewis et al (22). This software package was used for predicting target genes in mammalian species based on conservations across species and thermodynamic features of miRNA-target gene complexes (23). Target genes with a prediction score $>0.9$ were regarded as of high confidence.

Interaction network construction of target genes. Genes usually interact with each other to exert certain biological functions (24). Therefore, genes which interact with target genes were identified by the String database (25) and the interaction network of these genes was then established to broaden the understanding of their functions. The String database can rate the interactions from the aspects of homology, experiment and text mining.

Functional and pathway analyses. Functional and pathway analyses were performed for the genes in the network with
Table II. Details for the 4 differentially expressed miRNAs.

\begin{tabular}{lll}
\hline ID & p-value & $|\operatorname{logFC}|$ \\
\hline hsa-miR-146 & 0.005384 & 1.91716394 \\
hsa-miR-27 & 0.035685 & 1.49264032 \\
hsa-miR-877 & 0.039706 & 2.73696559 \\
hsa-miR-186 & 0.041463 & 1.1356551 \\
\hline
\end{tabular}

Expressing Analysis Systematic Explorer (EASE) (26) $(\mathrm{p}<0.05)$ which can identify the significant Gene Ontology (GO) terms and the genes that exist in the GO categories (27).

\section{Results}

Sequencing data. After the raw data were transformed into FASTQ format, the sequence read length was $36 \mathrm{bp}$. The number of original reads, miRNA alignments and miRNA types for each sample are listed in Table I. The expression levels of the miRNAs in each sample were then determined (data not shown).

Differentially expressed miRNAs. A total of 4 differentially expressed miRNAs were identified in the metastatic melanoma samples as compared with the primary cutaneous melanoma (control) samples according to the criteria $(\mathrm{p}<0.05$ and $|\log \mathrm{FC}|>1)$. These 4 miRNAs were hsa-miR-146, hsa-miR-27, hsa-miR-877 and hsa-miR-186. The information for these 4 miRNAs is presented in Table II.

Target genes and the interaction network. A total of 101 target genes with high confidence were found for hsa-miR-27 (data not shown). A total of 41 interactions (data not shown) were disclosed and the network was then constructed (Fig. 1).

Functional and pathway analyses of genes in the network. A total of 4 significant functional terms and 1 pathway were identified (Table III) for the genes in the interaction network. The most significant function was transcription, while the pathway of transcriptional misregulation in cancer was also revealed (Fig. 2). The genes which were enriched at the transcriptional level, included inhibitor of growth family, member 5 (ING5), 


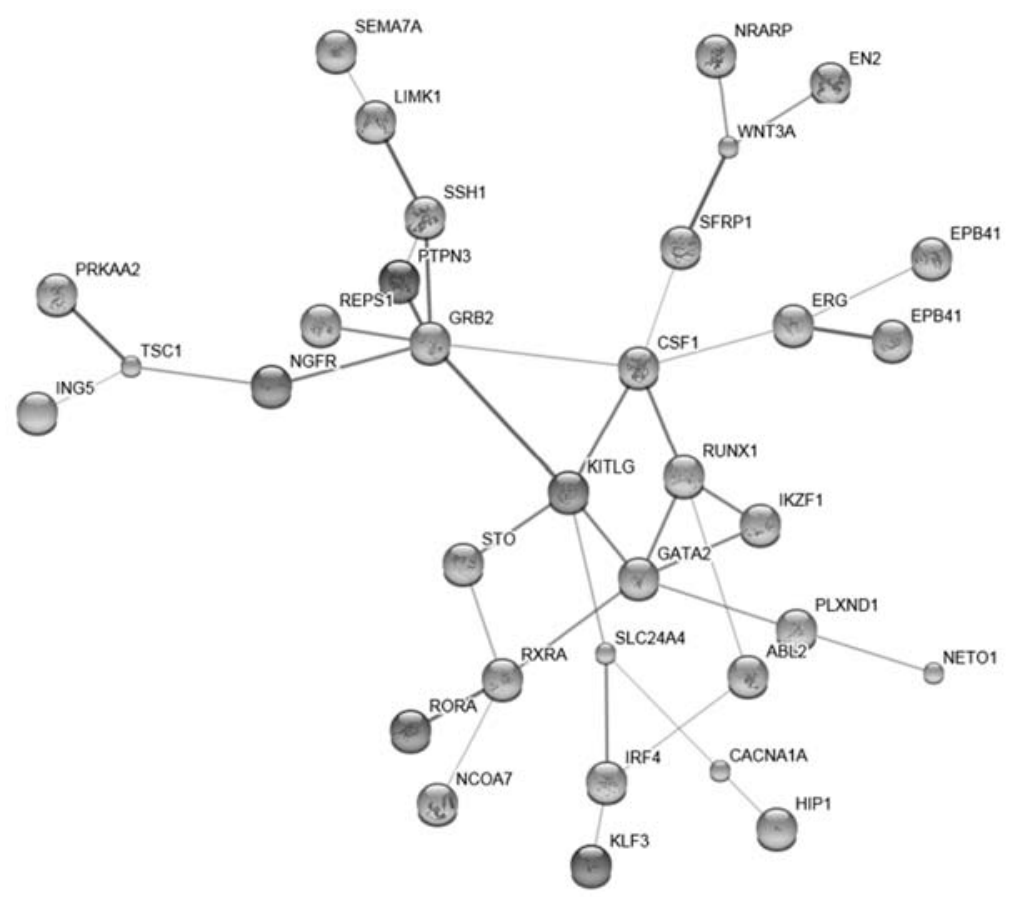

Figure 1. Interaction network of the target genes. A total of 101 target genes of has-miR-27 and 41 interactions were obtained. We then constructed a network based on these interactions.
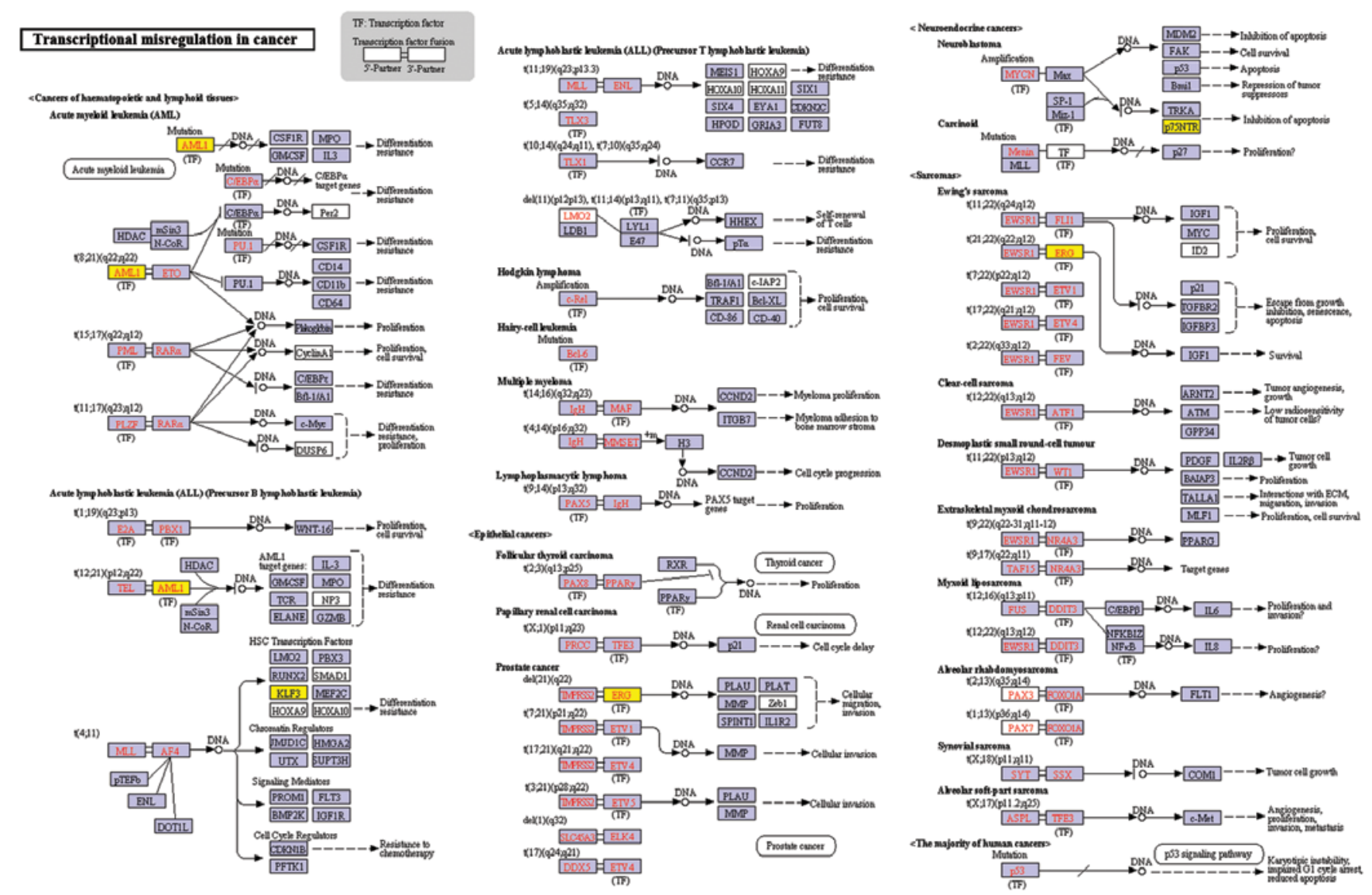

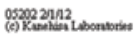

Figure 2. Pathways associated with enriched genes in the network. Genes in the yellow boxes are the target genes of differentially expressed hsa-miR-27.

GATA binding protein 2 (GATA2) and erythroblast transformation-specific (ETS)-related gene (ERG). Other genes, such as Ikaros family zinc finger 1 (IKZF1), interferon regulatory factor 4 (IRF4) and runt-related transcription factor 
Table III. Functional and pathway analyses of the genes in the network.

\begin{tabular}{lcl}
\hline Term - pathway & p-value & \multicolumn{1}{c}{ Genes } \\
\hline GO:0006350 - Transcription & 0.001954 & $\begin{array}{l}\text { ING5, GATA2, ERG, IKZF1, RXRA, NCOA7, } \\
\text { IRF4, RORA, RUNX1, HIP1, KLF3 }\end{array}$ \\
GO:0045449 - Regulation of transcription & 0.003148 & $\begin{array}{l}\text { ING5, GATA2, ERG, IKZF1, RXRA, NCOA7, } \\
\text { IRF4, EN2, RORA, RUNX1, HIP1, KLF3 }\end{array}$ \\
GO:0043228 - Non-membrane bounded organelle & 0.016522 & $\begin{array}{l}\text { GATA2, SSH1, PTPN3, EPB41, TSC1, IKZF1, } \\
\text { EPB41L4A, KITLG, IRF4, FGD6, ABL2, HIP1 } \\
\text { GO:0043232 - Intracellular non-membrane bounded organelle }\end{array}$ \\
$\begin{array}{ll}\text { GO:016522 } \\
\text { GATA2, SSH1, PTPN3, EPB41, TSC1, IKZF1, }\end{array}$ & $\begin{array}{l}\text { EPB41L4A, KITLG, IRF4, FGD6, ABL2, HIP1 } \\
\text { ERG, KLF3, AML1 }\end{array}$ \\
\hline
\end{tabular}

(RUNX), as well as retinoid $\mathrm{X}$ receptor, alpha (RXRA) and RAR-related orphan receptor A (RORA) were also included in the list (Table II).

\section{Discussion}

We identified 4 differentially expressed miRNAs by nextgeneration sequencing technology. In order to determine the functions of these miRNAs, the target genes of hsa-miR-27 were predicted and the interaction network of the genes was constructed. Finally, the functions of and pathways associated with these genes were analyzed in order to further elucidate the mechanisms responsible for metastatic melanoma.

The 4 differentially expressed miRNAs were hsa-miR-146, hsa-miR-27, hsa-miR-877 and hsa-miR-186. Rabinowits et al found the overexpression of hsa-miR-146 in lung cancer (28). Katakowski et al demonstrated that the expression of miR-146b-5p inversely correlated with glioma invasiveness in the brain (29). The study by Mertens-Talcott et al indicated that miR-27a is overexpressed in breast cancer cells, and that cell proliferation is decreased by the inhibition of this miRNA using antisense molecules in MDA-MB-231 cells (30). Scott et al reported that in SKBr3 breast cancer cells, the inhibitor of histone deacetylases, LAQ824, rapidly decreased miR-27a levels (31). miR-27a has also been shown to be upregulated in renal cell carcinoma as compared with normal kidneys (32) and acts as an oncogene in gastric adenocarcinoma (33). Guttilla and White further indicated that in breast cancer cells, miR-27a regulated the expression of FOXO1 (34), a putative tumor suppressor. The overexpression of miR- 877 has been reported in endometrial serous adenocarcinomas (35). In a previous study, when glyceollins were applied for the treatment of triple-negative breast cancer, the downregulation of miR-877 was observed (36). Baffa et al indicated that hsa-miR-186 was differentially expressed in paired primary and metastatic cancers (37). Leidinger et al demonstrated that hsa-miR-186 combined with other miRNAs was able to distinguish melanoma patients from healthy individuals (38). Therefore, we consider that these 4 miRNAs are potential targets for modulating the metastasis of melanoma cells.

To further elucidate the regulatory mechanisms, the target genes of the 4 miRNAs were predicted by TargetScan. A total of 101 target genes were revealed for hsa-miR-27. Functional enrichment analysis revealed that the most significantly enriched term was transcription, indicating that a number of gene expressions are regulated, which may lead to the acquisition of metastatic tumor cells. In addition, several target genes have been disclosed and are worthy of further investigation. ING5 is predicted to be one of the target genes which is linked to tumorigenesis in human head and neck carcinoma (39). GATA2 is a member of the GATA family of zinc-finger transcription factors. Bohm et al found that the high expression of GATA2 is associated with metastatic progression and biochemical recurrence in prostate cancer through the regulation of key androgen-regulated genes (40). ERG is a member of the ETS family and a number of studies have investigated its role in prostate cancer $(41,42)$.

Overall, next-generation sequencing technology was adopted in this study to identify the differentially expressed miRNAs in metastatic melanoma as compared with primary cutaneous melanoma samples. Moreover, the functions and pathways of target genes were revealed, which may provide insight into the regulatory mechanism. The data presented in our study may prove beneficial in the diagnosis and treatment of metastatic melanoma and may aid in the development of novel clinical applications using miRNAs. However, further sutdies are required to confirm our results.

\section{Acknowledgements}

This study was supported by a grant from the National Natural Science Foundation of China (no. 30900767 to Jianglin Zhang). The authors thank Professor Ming Zhou for providing insightful comments.

\section{References}

1. Hernandez BY, Green MD, Cassel KD, Pobutsky AM, Vu V and Wilkens LR: Preview of Hawaii cancer facts and figures 2010. Hawaii Med J 69: 223-224, 2010.

2. Gray-Schopfer V, Wellbrock C and Marais R: Melanoma biology and new targeted therapy. Nature 445: 851-857, 2007.

3. Hatfield S and Ruohola-Baker H: microRNA and stem cell function. Cell Tissue Res 331: 57-66, 2008.

4. Agarwala SS: Current systemic therapy for metastatic melanoma. Expert Rev Anticancer Ther 9: 587-595, 2009. 
5. Calin GA and Croce CM: MicroRNA-cancer connection: the beginning of a new tale. Cancer Res 66: 7390-7394, 2006.

6. Iorio MV,Ferracin M, Liu CG, et al: MicroRNA gene expression deregulation in human breast cancer. Cancer Res 65: 7065-7070, 2005 .

7. Ma L, Teruya-Feldstein J and Weinberg RA: Tumour invasion and metastasis initiated by microRNA-10b in breast cancer. Nature 449: 682-688, 2007.

8. Yanaihara N, Caplen N, Bowman E, et al: Unique microRNA molecular profiles in lung cancer diagnosis and prognosis. Cancer Cell 9: 189-198, 2006.

9. Zhou J, Yu L, Gao X, et al: Plasma microRNA panel to diagnose hepatitis B virus-related hepatocellular carcinoma. J Clin Oncol 29: 4781-4788, 2011

10. Chen CZ: MicroRNAs as oncogenes and tumor suppressors. N Engl J Med 353: 1768-1771, 2005.

11. Hatfield SD, Shcherbata HR, Fischer KA, Nakahara K Carthew RW and Ruohola-Baker H: Stem cell division is regulated by the microRNA pathway. Nature 435: 974-978, 2005.

12. Giles KM, Barker A, Zhang PM, Epis MR and Leedman PJ: MicroRNA regulation of growth factor receptor signaling in human cancer cells. Methods Mol Biol 676: 147-163, 2011.

13. Jiang S, Zhang HW, Lu MH, et al: MicroRNA-155 functions as an OncomiR in breast cancer by targeting the suppressor of cytokine signaling 1 gene. Cancer Res 70: 3119-3127, 2010.

14. Shumway M, Cochrane G and Sugawara H: Archiving next generation sequencing data. Nucleic Acids Res 38: D870-D871, 2010.

15. Cock PJ, Fields CJ, Goto N, Heuer ML and Rice PM: The Sanger FASTQ file format for sequences with quality scores, and the Solexa/Illumina FASTQ variants. Nucleic Acids Res 38 $1767-1771,2010$

16. Kaminuma E, Mashima J, Kodama Y, et al: DDBJ launches a new archive database with analytical tools for next-generation sequence data. Nucleic Acids Res 38: D33-D38, 2010.

17. Langmead B, Trapnell C, Pop M and Salzberg SL: Ultrafast and memory-efficient alignment of short DNA sequences to the human genome. Genome Biol 10: R25, 2009.

18. Trapnell C and Salzberg SL: How to map billions of short reads onto genomes. Nat Biotechnol 27: 455-457, 2009.

19. Friedlander MR, Chen W, Adamidi C, et al: Discovering microRNAs from deep sequencing data using miRDeep. Nat Biotechnol 26: 407-415, 2008.

20. Buermans HP, Ariyurek Y, van Ommen G, den Dunnen JT and 't Hoen PA: New methods for next generation sequencing based microRNA expression profiling. BMC Genomics 11: 716, 2010.

21. Smyth GK: Limma: linear models for microarray data In: Bioinformatics and Computational Biology Solutions using $\mathrm{R}$ and Bioconductor. Gentleman R, Carey V, Dudoit S, Irizarry R and Huber W (eds). Springer, New York, pp397-420, 2005.

22. Lewis BP, Burge CB and Bartel DP: Conserved seed pairing, often flanked by adenosines, indicates that thousands of human genes are microRNA targets. Cell 120: 15-20, 2005

23. Friedman RC, Farh KK, Burge CB and Bartel DP: Most mammalian mRNAs are conserved targets of microRNAs. Genome Res 19: 92-105, 2009.

24. Tong AH, Drees B, Nardelli G, et al: A combined experimental and computational strategy to define protein interaction networks for peptide recognition modules. Science 295: 321-324, 2002.
25. Szklarczyk D, Franceschini A, Kuhn M, et al: The STRING database in 2011: functional interaction networks of proteins, globally integrated and scored. Nucleic Acids Res 39: D561-D568, 2011.

26. Hosack DA, Dennis G Jr, Sherman BT, Lane HC and Lempicki RA: Identifying biological themes within lists of genes with EASE. Genome Biol 4: R70, 2003.

27. Steeg PS: Tumor metastasis: mechanistic insights and clinical challenges. Nat Med 12: 895-904, 2006.

28. Rabinowits G, Gercel-Taylor C, Day JM, Taylor DD and Kloecker GH: Exosomal microRNA: a diagnostic marker for lung cancer. Clin Lung Cancer 10: 42-46, 2009.

29. Katakowski M, Zheng X, Jiang F, Rogers T, Szalad A and Chopp M: MiR-146b-5p suppresses EGFR expression and reduces in vitro migration and invasion of glioma. Cancer Invest 28: $1024-1030,2010$

30. Mertens-Talcott SU, Chintharlapalli S, Li X and Safe S: The oncogenic microRNA-27a targets genes that regulate specificity protein transcription factors and the G2-M checkpoint in MDA-MB-231 breast cancer cells. Cancer Res 67: 11001-11011, 2007.

31. Scott GK, Mattie MD, Berger CE, Benz SC and Benz CC: Rapid alteration of microRNA levels by histone deacetylase inhibition. Cancer Res 66: 1277-1281, 2006

32. Gottardo F, Liu CG, Ferracin M, et al: Micro-RNA profiling in kidney and bladder cancers. Urol Oncol 25: 387-392, 2007.

33. Liu T, Tang H, Lang Y, Liu M and Li X: MicroRNA-27a functions as an oncogene in gastric adenocarcinoma by targeting prohibitin. Cancer Lett 273: 233-242, 2009.

34. Guttilla IK and White BA: Coordinate regulation of FOXO1 by miR-27a, miR-96, and miR-182 in breast cancer cells. J Biol Chem 284: 23204-23216, 2009.

35. Hiroki E, Akahira J, Suzuki F, et al: Changes in microRNA expression levels correlate with clinicopathological features and prognoses in endometrial serous adenocarcinomas. Cancer Sci 101: 241-249, 2010

36. Rhodes LV, Tilghman SL, Boue SM, et al: Glyceollins as novel targeted therapeutic for the treatment of triple-negative breast cancer. Oncol Lett 3: 163-171, 2012.

37. Baffa R, Fassan M, Volinia S, et al: MicroRNA expression profiling of human metastatic cancers identifies cancer gene targets. J Pathol 219: 214-221, 2009.

38. Leidinger P, Keller A, Borries A, et al: High-throughput miRNA profiling of human melanoma blood samples. BMC Cancer 10: 262,2010

39. Li X, Nishida T, Noguchi A, et al: Decreased nuclear expression and increased cytoplasmic expression of ING5 may be linked to tumorigenesis and progression in human head and neck squamous cell carcinoma. J Cancer Res Clin Oncol 136: 1573-1583, 2010.

40. Bohm M, Locke WJ, Sutherland RL, Kench JG and Henshall SM A role for GATA-2 in transition to an aggressive phenotype in prostate cancer through modulation of key androgen-regulated genes. Oncogene 28: 3847-3856, 2009.

41. Wang J, Cai Y, Ren C and Ittmann M: Expression of variant TMPRSS2/ERG fusion messenger RNAs is associated with aggressive prostate cancer. Cancer Res 66: 8347-8351, 2006.

42. Carver BS, Tran J, Gopalan A, et al: Aberrant ERG expression cooperates with loss of PTEN to promote cancer progression in the prostate. Nat Genet 41: 619-624, 2009. 